the steatosis would cause swelling of the hepatocytes which could then further compromise the blood supply. This hypothesis is unlikely in this case as over $90 \%$ of the liver capsule was affected.

I am grateful to Dr $S$ Waldeck for permission to report his patient.
1 Grove A, Vyberg B, Vyberg $M$. Focal fatty change of the liver. Virchows Arch (Pathol Anat) 1991;419: 69-75.

2 Wanless I, Bargman J, Oreopoulos D, Vas S. Subcapsular steatonecrosis in response to peritoneal insulin delivery: a clue to the pathogenesis of steatonecrosis in obesity. Mod Pathol 1989;2:69-74.

3 Brawer M, Austin G, Lewin K. Focal fatty change of the liver, a hitherto poorly recognized entity. Gastroenterol 1980;78:247-52.

\title{
Immunohistochemical and in situ hybridisation detection of adenovirus early region 1A (E1A) gene in the microglia of human brain tissue
}

\author{
T Matsuse, Y Namba, K Ikeda, S Inoue, T Hosoi, Y Ouchi, Y Fukuchi, H Orimo
}

Department of

Geriatrics, Faculty of

Medicine, University

of Tokyo

T Matsuse

$S$ Inoue

T Hosoi

Y Ouchi

Y Fukuchi

H Orimo

Department of

Neuropathology,

Institute of Brain

Research, Faculty of

Medicine, University

of Tokyo

Y Namba

Department of

Ultrastructure and

Histochemistry,

Psychiatric Research

Institute of Tokyo

K Ikeda

Correspondence to:

Dr Takeshi Matsuse,

Department of Geriatrics,

Faculty of Medicine,

University of Tokyo, 7-3-1

Hongo Bunkyo-ku, Tokyo

113, Japan.

Accepted for publication

7 September 1993

\begin{abstract}
To investigate whether brain tissue is infected latently by adenovirus via a monocyte/microglia-mediated entry mechanism, brain tissue resected at necropsy from seven senile subjects (five with senile dementia of Alzheimer type (SDAT) and two subjects without pathological changes) was examined for adenovirus early region $1 \mathrm{~A}$ (E1A) gene and its expression using in situ hybridisation and immunohistochemical staining. HLA-DR positive, reactive microglial cells in both SDAT and normal brain tissue showed positive hybridisation and immunoreactive expression of adenovirus E1A. Thus there may be monocyte/ microglia-mediated entry of adenovirus in the central nervous system which would be a novel and presumably common interaction between brain tissue and adenovirus.
\end{abstract}

(F Clin Pathol 1994;47:275-277)

Adenoviruses are common; several of the 41 subtypes of adenovirus cause respiratory infections in man. Group C serotypes $(1,2,5$ and 6) of adenovirus are of particular interest because they are known to reside latently in the tonsils ${ }^{1}$ and peripheral blood mononuclear cells such as lymphocytes ${ }^{2}$ for many years after infection.

The origin of the microglia has long been a subject of debate, but it now seems clear that monocytes enter the normal central nervous system and follow a series of morphological transformations as they differentiate into microglia. As monocytes migrate into the normal central nervous system, they represent potential vehicles for the entry of pathogens into the nervous system. Two members of the lentiviruses, Maedi-Visna (MV) virus and HIV, use the monocyte-mediated ingress mechanism, the so-called "Trojan horse" mechanism of central nervous system invasion, and persistently infect microglia. ${ }^{3}$

The protein products of adenovirus early region $1 \mathrm{~A}(\mathrm{E} 1 \mathrm{~A})$ gene have a role in the activation of other early promotors, as well as transcription from several host promotors. Furthermore, the E1A proteins are essential for the transformation of cells by adenovirus. ${ }^{4}$

The purpose of this study was to examine human senile brain tissues to determine whether those microglia are infected latently by adenovirus. We particularly used senile dementia of Alzheimer type (SDAT) brain tissues because there are abundant reactive microglial cells in the brains of those patients. $^{5}$

\section{Methods}

Five post mortem human brains from patients with a clinicohistopathological diagnosis of SDAT (age 73-93 years, mean 85) and two senile brains without any pathological changes (patients aged 77 and 76 years) were investigated.

Brain tissue obtained at necropsy was snap frozen and stored at $-80^{\circ} \mathrm{C}$. Cryostat sections were fixed in cold acetone and subjected to a combination of in situ hybridisation with digoxigenine-11-dUTP-labelled probe for the E1A viral genome and immunohistochemical staining with HLA-DR monoclonal antibody (two SDAT and one normal brains). Briefly, sections were mounted on coated glass slides and acetylated. The sections were incubated with a hybridisation mixture containing digoxigenine-labelled probe for the E1A viral genome. A hybridisation mixture containing labelled PBR322 (an unrelated adenovirus probe) served as a control. The probe DNA for the E1A gene was a 756 base pair fragment of the E1A region of adenovirus type 5 which had been subcloned into pUC13 (gift of JC Hogg, Vancouver, BC, Canada). ${ }^{6}$ The 
DNA was first denatured by heating the slides at $90^{\circ} \mathrm{C}$, then allowed to hybridise at $37^{\circ} \mathrm{C}$ overnight. After washing off the unbound probe, the sections were incubated with alkaline phosphatase conjugated antidigoxigenine antibody and subjected to BCIP-NBT development. Following the hybridisation procedure, the sections were subjected to immunostaining using a mouse monoclonal antibody to HLA-DR (Dako, Denmark), a marker used for human reactive

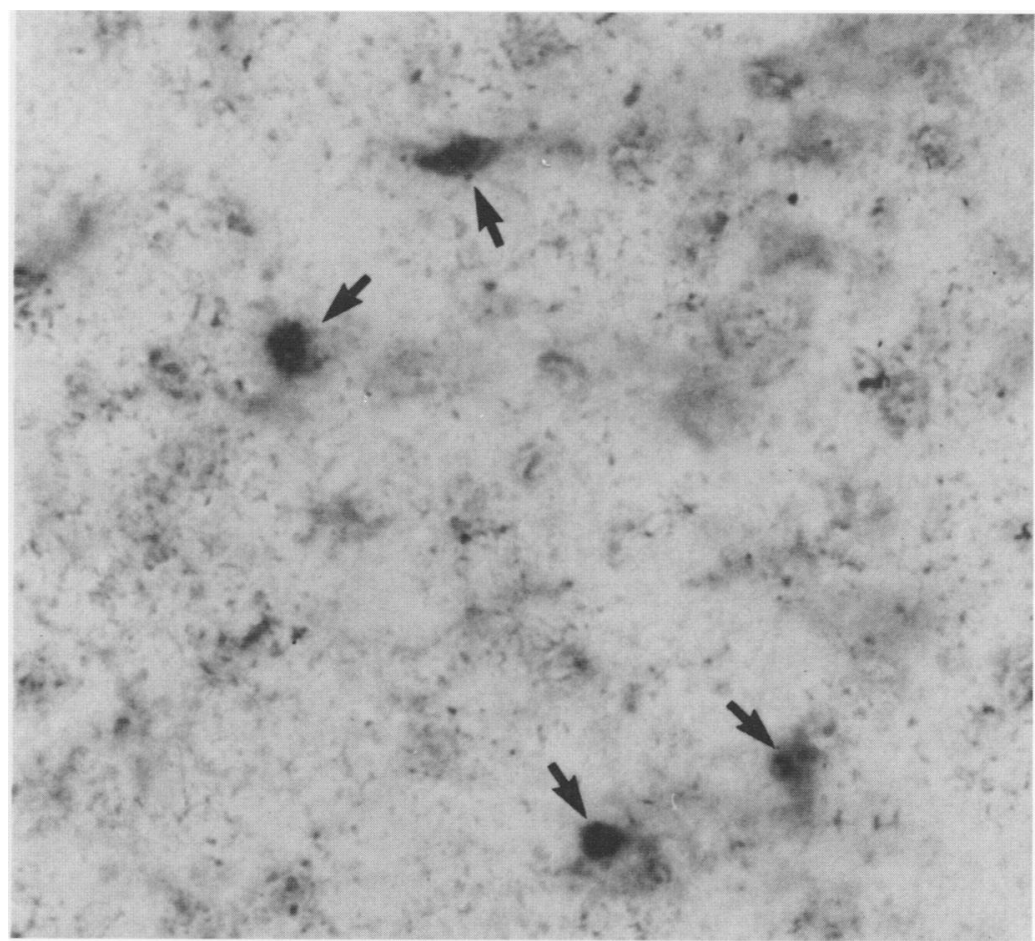

Figure 1 Photomicrograph from an ISH study of a SDAT brain where positive signals for $E 1 A$ (arrows) were seen in $H L A-D R$ positive cells (brown staining).

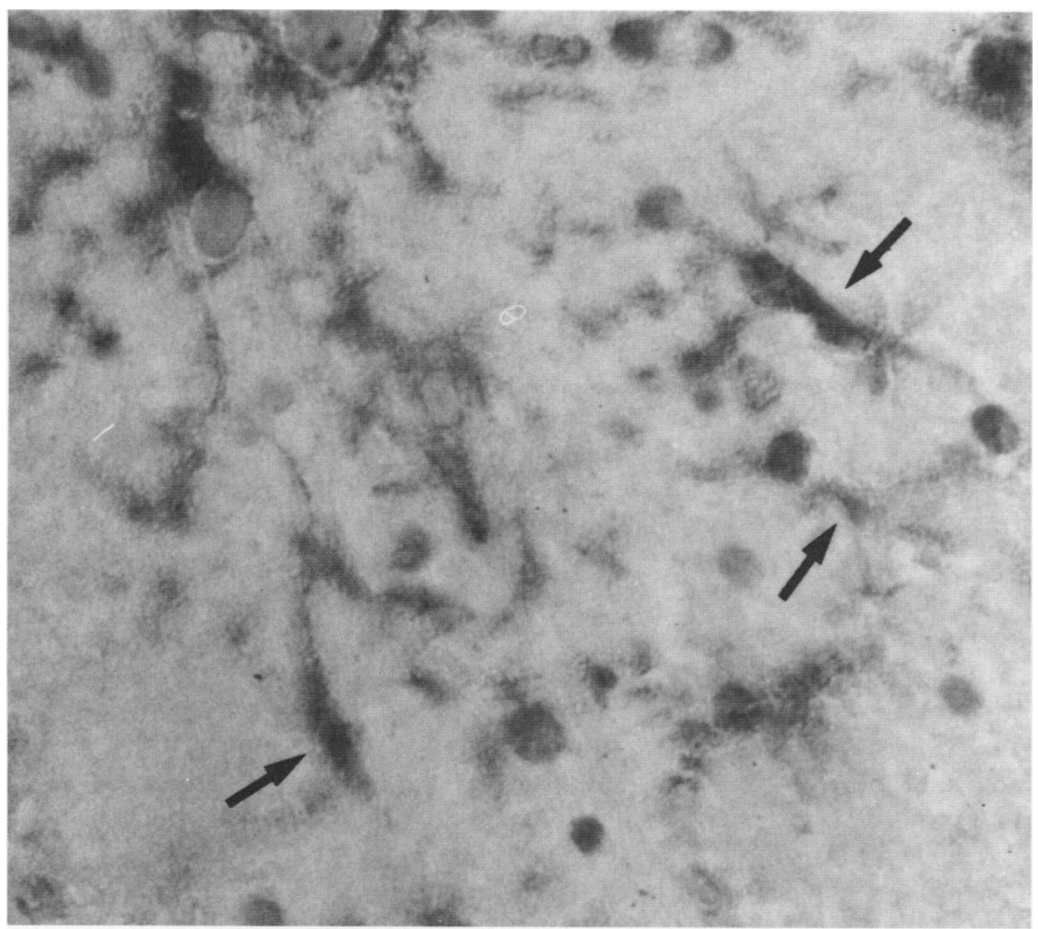

Figure 2 E1A immunoreactive cells (arrows) in brain tissue from a patient with SDAT. microglial cells. ${ }^{5}$ After incubation in peroxidase conjugated streptavidin, reaction products were visualised using diaminobenzidine tetrahydrochloride (DAB).

Immunohistochemical studies for the E1A protein were performed with the standard avidin-biotin complex (ABC) method. The primary antibody (a mouse monoclonal antibody to E1A protein of adenovirus type 5) was purchased from Oncogenescience, Manhasset, NY, USA. The sections were blocked with $10 \%$ fetal calf serum, before incubation overnight with $1 \mu \mathrm{g} / \mathrm{ml}$ of primary antibody, and then washed in phosphate buffered saline. A biotinylated anti-rabbit immunoglobulin was applied before incubation with peroxidase conjugated streptavidin; reaction products were visualised using DAB. Double immunostaining studies were also conducted for the E1A product and HLADR. After the sections were immunostained with the monoclonal antibody to the E1A protein (as above), sections were washed in phosphate buffered saline and blocked with fetal calf serum, and incubated in the monoclonal antibody to HLA-DR (Dako). After incubation in alkaline phosphatase conjugated streptavidin, sections were subjected to BCIP-NBT development.

\section{Results}

We first examined cryostat sections from three brains (two SDAT and one normal) by in situ hybridisation histochemistry using the probe that covers the E1A genomic region of adenovirus type 5. Hybridisation positive signals were seen in many cells in various parts of the grey and white matter of all the brain tissue. We also combined in situ hybridisation histochemistry with immunostaining using antibody to the glial cell marker. The cells with the hybridisation signal for the E1A probe were all positive for the class II major histocompatibility antigen (HLA-DR), a marker used for human reactive microglial cells (fig 1 ). ${ }^{5}$

We then wanted to determine whether the E1A gene was expressed in these microglial cells. Scattered cells in all sections from both SDAT and normal brains were positive for the E1A protein by immunostaining (fig 2). Morphologically they were consistent with microglial cells. To confirm this, a double immunostaining study with antibodies to the E1A protein and HLA-DR was conducted. This study clearly showed co-localisation of the E1A and HLA-DR immunoreactivities. Thus it is clear that a certain number of reactive microglial cells in both normal senile brains and SDAT brains harbor the E1A gene and that it is expressed in these cells.

\section{Discussion}

Some conventional viruses cause acute encephalitis but they are usually eliminated by host immune systems. However, measles and rubella viruses are known to persist in a 
latent form in the brain. ${ }^{7}$ It has also been reported that a fragment of herpes simplex type 1 DNA was found in human brain tissue. ${ }^{8}$ The data of the present study show a novel, and presumably widely occurring, interaction of brain tissue and adenovirus. While adenovirus causes common respiratory infections, it is unlikely that the virus brings about a latent infection in brain tissue. Given that brain microglia are derived from blood monocytes and there is continuous supply of monocytes from circulation into brain parenchyma even in adults, we speculate that the E1A positive microglia could be derived from the blood monocytes that are latently infected with adenovirus, as occurs in peripheral lymphocytes. ${ }^{2}$ Such a monocyte/ microglia-mediated ingress of viruses into the central nervous system has been reported for HIV. ${ }^{3}$

Epidemiological studies of Japanese subjects over 30 years of age showed neutralising antibodies to adenovirus types 1 and 2 in $95 \%$ of the population, and antibodies to adenovirus types 5 and 6 in around $60 \%$ of the population. Taken together with the report that 13 out of 17 samples of human lymphocytes were positive for adenovirus DNA, ${ }^{2}$ it is possible that circulating blood cells in the Japanese population, and presumably other populations, may be latently infected with adenovirus, and this is widespread. This hypothesis could explain the presence of the E1A protein positive microglia in all seven brains studied here.

E1A proteins interact with the product of the retinoblastoma gene. ${ }^{9}$ The retinoblastoma protein may act as a growth inhibitory factor based on its ability to control transcription by binding to transcription factors. ${ }^{9}$ A recent hypothesis suggests that the binding of E1A to retinoblastoma protein displaces the protein from these transcription factors, allowing cell division and tissue growth to proceed. ${ }^{9}$ Our findings raise the interesting possibility that the expression of the E1A gene present in human brain microglia may activate microglia at the transcription level and function in activating the central nervous system immune response, or may alter the homeostatic balance between microglia and other glia or microglia and neurons, although further work is needed to clarify this.

We thank Dr S Hayashi and Professor JC Hogg for cloned plasmid DNA. We also thank Dr MD Fetters for helpful comments.

1 Green M, Wold WSM, Mackey JK, Rigden P. Analysis of human tonsil and cancer DNAs and RNAs for DNA sequences of group C (serotypes 1, 2, 5 and 6) human
adenoviruses. Proc Natl Acad Sci USA 1979; adenoviruses.

2 Horvath J, Palkonyay L, Weber J. Group C adenovirus DNA sequences in human lymphoid cells. $\mathcal{f}$ Virol 1986;59:189-92.

3 Williams AE, Blakemore WF. Annotation. Monocytemediated entry of pathogens into the central nervous system. Neuropathol Appl Neurobiol 1990;16:377-92.

4 Shenk T, Flint J. Transcriptional and transforming activities of the adenovirus E1A proteins. Adv Cancer Res 1991;57:47-85.

5 McGeer PL, Itagaki S, Tago H, McGeer EG. Reactive microglia in patients with senile dementia of the Alzheimer type are positive for the histocompatibility Alzheimer type are positive for the histocompatibility
glycoprotein HLA-DR. Neurosci Lett 1987;79:195-200.

6 Matsuse T, Hayashi S, Kuwano K, Keunecke H, Jefferies WA, Hogg JC. Latent adenovirus infection in the pathogenesis of chronic airways obstruction. Am Rev Respir Dis 1992;146:177-84.

7 Johnson RT. Part III: chronic neurological diseases. Viral infections of the nervous system. New York: Raven Press, 1982:201-310.

8 Fraser NW, Lawrence WC, Wroblewska Z, Gilden DH, Koprowski H. Herpes simplex type 1 DNA in human brain tissue. Proc Natl Acad Sci USA 1981;78:6461-5.

9 Bandara LR, LaThangue NB. Adenovirus Ela prevents retinoblastoma gene product from complexing with cellular transcription factor. Nature 1991;351:494-7. 